Homology, Homotopy and Applications, vol.9(1), 2007, pp.163-183

\title{
ON LIFTING STABLE DIAGRAMS IN FROBENIUS CATEGORIES
}

\author{
MATTHIAS KÜNZER
}

(communicated by Claude Cibils)

\begin{abstract}
Suppose given a Frobenius category $\mathcal{E}$, i.e. an exact category with a big enough subcategory $\mathcal{B}$ of bijectives. Let $\mathcal{E}:=\mathcal{E} / \mathcal{B}$ denote its classical stable category. For example, we may take $\mathcal{E}$ to be the category of complexes $\mathrm{C}(\mathcal{A})$ with entries in an additive category $\mathcal{A}$, in which case $\mathcal{E}$ is the homotopy category of complexes $\mathrm{K}(\mathcal{A})$. Suppose given a finite poset $D$ that satisfies the combinatorial condition of being ind-flat. Then, given a diagram of shape $D$ with values in $\mathcal{E}$ (i.e. stably commutative), there exists a diagram consisting of pure monomorphisms with values in $\mathcal{E}$ (i.e. commutative) that is isomorphic, as a diagram with values in $\underline{\mathcal{E}}$, to the given diagram.
\end{abstract}

Dedicated to Claus M. Ringel on the occasion of his 60th birthday.

\section{Contents}

0 Introduction $\quad \mathbf{1 6 4}$

0.1 The problem . . . . . . . . . . . . . . . . . . . . . . 164

0.2 Problems that remain open . . . . . . . . . . . . . . . 164

0.3 Motivation . . . . . . . . . . . . . . . . . . 165

0.4 Result . . . . . . . . . . . . . . . . . . . . 165

0.5 Notation and conventions . . . . . . . . . . . . . . . . 166

1 Limits and pure monomorphisms $\quad 167$

1.1 Crowns . . . . . . . . . . . . . . . . . 167

1.2 Limits . . . . . . . . . . . . . . . . . . . 170

2 Replacement lemmata $\quad 172$

2.1 Replacement . . . . . . . . . . . . . . . . . . . . . 172

2.2 A purely monomorphic replacement . . . . . . . . . . . . 173

2.3 A replacement that adds a commutativity . . . . . . . . . . . . 174

3 Density

Received September 3, 2005, revised October 11, 2006; published on January 16, 2007.

2000 Mathematics Subject Classification: 18E10.

Key words and phrases: stable Frobenius category.

Copyright (C) 2007, International Press. Permission to copy for private use granted. 


\section{Introduction}

\subsection{The problem}

Let $\mathcal{E}$ be a Frobenius category; that is, an exact category in the sense of QUILLEN $[\mathbf{9}, \S 2]$ with enough bijective objects; cf. e.g. [6, Sec. A.6]. Let $\mathcal{B} \subseteq \mathcal{E}$ denote the full subcategory of bijective objects, and let $\underline{\mathcal{E}}=\mathcal{E} / \mathcal{B}$ denote the classical stable

category of $\mathcal{E}$. Let $\mathcal{E}^{\text {mono }} \subseteq \mathcal{E}$ denote the subcategory of pure monomorphisms of $\mathcal{E}$. Write $\mathcal{E} \stackrel{N}{\longrightarrow} \underline{\mathcal{E}}$ for the residue class functor, and likewise, by abuse of notation, $\mathcal{E}^{\text {mono }} \stackrel{N}{\longrightarrow} \mathcal{E}$ for its restriction to $\mathcal{E}^{\text {mono }}$.

Let $D$ be a category. A functor $X$ from $D$ to $\mathcal{E}$ is a diagram of shape $D$ with values in $\underline{\mathcal{E}}$, sometimes called a stable diagram. Choosing representatives in $\mathcal{E}$, we may think of $X$ as a "diagram of shape $D$ with values in $\mathcal{E}$, that stably commutes". We ask under which conditions on $D$ we can find a "strictly commutative" diagram $X^{\prime}$ of shape $D$ with values in $\mathcal{E}$ that becomes isomorphic to the "stably commutative" diagram $X$, when both are considered in the category of diagrams of shape $D$ with values in $\underline{\mathcal{E}}$.

Put formally, the residue class functor $\mathcal{E}^{\text {mono }} \stackrel{N}{\longrightarrow} \underline{\mathcal{E}}$ induces a functor $\mathcal{E}^{\text {mono }}(D) \stackrel{N(D)}{\longrightarrow} \underline{\mathcal{E}}(D)$ on the diagrams of shape $D$ by pointwise application. We ask for a sufficient condition on $D$ for $\mathcal{E}^{\text {mono }}(D) \stackrel{N(D)}{\longrightarrow} \underline{\mathcal{E}}(D)$ to be dense for all Frobenius categories $\mathcal{E}$; that is, for its induced map on the isoclasses to be surjective.

Such a condition is then a fortiori sufficient for the induced functor $\mathcal{E}(D) \stackrel{N(D)}{\longrightarrow} \underline{\mathcal{E}}(D)$ to be dense. It turns out to be technically advantageous to consider $\mathcal{E}^{\text {mono }}$ instead of $\mathcal{E}$.

Restricting ourselves to the case of $D$ being a finite poset, we will find a sufficient condition in combinatorial terms on $D$ ensuring that $\mathcal{E}^{\text {mono }}(D) \stackrel{N(D)}{\longrightarrow} \mathcal{E}(D)$ is dense, called ind-flatness; cf. Section 0.4 below.

\subsection{Problems that remain open}

0.2.1. An obstruction to the Density of $N(D)$ ?

I do not know a necessary and sufficient combinatorial condition on $D$ for $\mathcal{E}^{\text {mono }}(D) \stackrel{N(D)}{\longrightarrow} \underline{\mathcal{E}}(D)$ to be dense for all Frobenius categories $\mathcal{E}$. For instance, it is dense for $D=\Delta_{m} \times \Delta_{n}$, where $m, n \geqslant 0$. However, I do not know whether it is dense for $D=\Delta_{1} \times \Delta_{1} \times \Delta_{1}$.

Considering a category of spaces instead of a Frobenius category $\mathcal{E}$, DWYER, KAN and SMITH have exhibited classes in certain Hochschild-Mitchell cohomology groups of $D$ in dimension $\geqslant 3$ that are obstructions to the density of the analogue of $N(D)$; cf. $[2,3.5,3.6]$.

MiTCHELL gave a combinatorial criterion for the Hochschild-Mitchell cohomology groups to vanish in dimensions $\geqslant 3$; cf. [8, Th. 35.7]; cf. Section 5. This criterion is 
fulfilled by $\Delta_{1} \times \Delta_{1}$, but not by $\Delta_{1} \times \Delta_{1} \times \Delta_{1}$.

I do not know whether ind-flat finite posets satisfy Mitchell's criterion. I do not know whether there exists an obstruction theory in the spirit of [2] for Frobenius categories. If both should turn out to be true, this would yield the "true reason" for density in the case of an ind-flat finite poset. And if, moreover, the obstruction classes should turn out to be calculable for $D=\Delta_{1} \times \Delta_{1} \times \Delta_{1}$, it would probably also yield an example in which density fails.

\subsubsection{1-EPIMORPHY?}

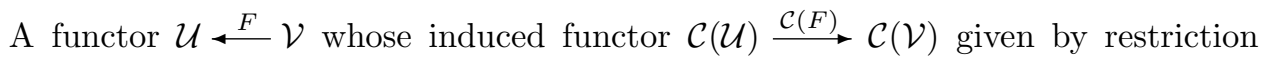
along $F$ is full and faithful for all categories $\mathcal{C}$ is called 1 -epimorphic; cf. [6, Sec. A.8]. If the finite poset $D$ is a finite quasi-tree in the sense of Definition 4.1, then $\mathcal{E}^{\text {mono }}(D)$ $\stackrel{N(D)}{\longrightarrow} \mathcal{E}(D)$ is 1-epimorphic; see Proposition 4.4. We do not know any less drastically restrictive sufficient condition on $D$ for this 1-epimorphy to hold.

\subsection{Motivation}

The functor $\mathcal{E}^{\text {mono }}\left(\Delta_{1}\right) \stackrel{N\left(\Delta_{1}\right)}{\longrightarrow} \underline{\mathcal{E}}\left(\Delta_{1}\right)$ being dense can be seen as the technical reason why every morphism in $\underline{\mathcal{E}}$ can be extended to a distinguished triangle in the sense of VERDIER [10], while the functor $\mathcal{E}^{\text {mono }}\left(\Delta_{2}\right) \stackrel{N\left(\Delta_{2}\right)}{\longrightarrow} \underline{\mathcal{E}}\left(\Delta_{2}\right)$ being dense can be seen as the main technical reason why the octahedral axiom (TR 4) of loc. cit. holds. We attempt to extend this density property as far as possible.

Heller asked the density question in a more general setting; cf. [4, p. 4; Prop. III.3.9 and remark thereafter]. This question also appeared in the discussion of the axioms of a triangulated dérivateur, due to Grothendieck and Maltsiniotis; cf. [7, p. 4]; cf. [5], [3].

For applications in topology of the solution of an analogous problem for spaces, see $[\mathbf{1}$, Sec. 2].

\subsection{Result}

Let $Q$ be a finite poset, considered as a category. For $q \in Q$, let

$$
\begin{aligned}
\Lambda(q) & :=\{r \in Q: r \leqslant q\} \\
\Lambda^{0}(q) & :=\{r \in Q: r<q\} \\
\mathrm{V}(q) & :=\{r \in Q: r \geqslant q\} \\
\max (Q) & :=\{r \in Q: \mathrm{V}(r)=\{r\}\} \\
\text { Obind-crown }(Q) & :=\bigcup_{r, s \in \max (Q)} \operatorname{Obmax}(\Lambda(r) \cap \Lambda(s)),
\end{aligned}
$$

yielding a poset ind-crown $(Q)$ via

$$
r<_{\text {ind-crown }(Q)} s \quad: \Longleftrightarrow r<_{Q} s \text { and } r \notin \max (Q) \text { and } s \in \max (Q) .
$$


We sketch a finite poset $Q$ and its ind-crown.

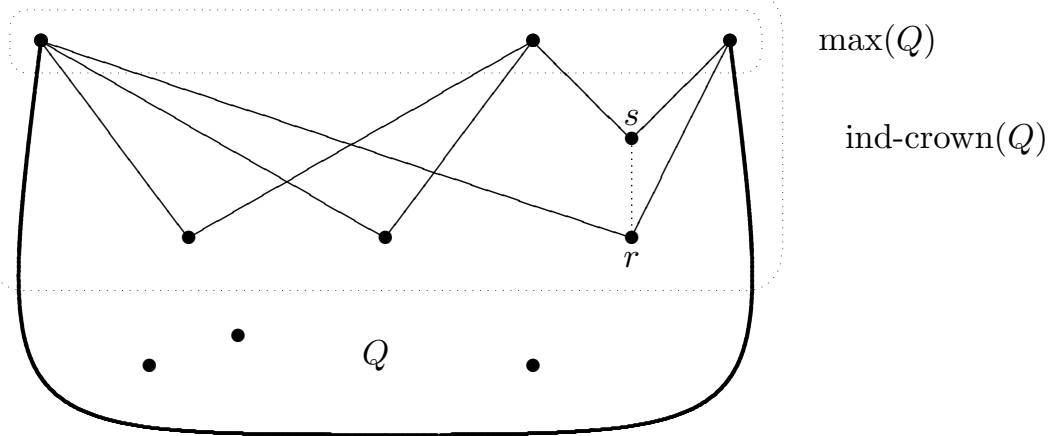

While it might be the case that $r<s$ in $Q$, we have $r \nless s$ in ind-crown $(Q)$ since $s \notin \max (Q)$.

A finite poset $P$ is called ind-flat if ind-crown $\left(\Lambda^{0}(p)\right)$ is componentwise 1-connected for each $p \in P$; cf. Definition 1.2. For some examples, see Definition 2.1 and Example 2.2.

Theorem (Theorem 3.1). Suppose given an ind-flat finite poset $D$ and a Frobenius category $\mathcal{E}$. Then $\mathcal{E}^{\text {mono }}(D) \stackrel{N(D)}{\longrightarrow} \mathcal{E}(D)$ is dense.

\section{Acknowledgements}

I thank the referee for pointing out the work of DwYER, KAN and SMITH [2].

\subsection{Notation and conventions}

(i) For $a, b \in \mathbf{Z}$, we denote by $[a, b]:=\{z \in \mathbf{Z}: a \leqslant z \leqslant b\}$ the integral interval.

(ii) Given $n \geqslant 0$, we let $\Delta_{n}$ be the linearly ordered set [0,n], with ordering inherited from $\mathbf{Z}$.

(iii) Given a set $M$, we denote by $\mathfrak{P}(M)=\{N: N \subseteq M\}$ its power set. If $M$ is finite, then $\# M$ denotes the cardinality of $M$.

(iv) All categories are supposed to be small with respect to a sufficiently big universe.

(v) Composition of morphisms is written on the right, $\stackrel{a}{\longrightarrow} \stackrel{b}{\longrightarrow}=\stackrel{a b}{\longrightarrow}$.

(vi) The category of functors and transformations from a category $D$ to a category $\mathcal{C}$ is denoted by $\llbracket D, \mathcal{C} \rrbracket$, or by $\mathcal{C}(D)$. The latter is used to emphasise that the objects of $\mathcal{C}(D)$ can be viewed as diagrams of shape $D$ with values in $\mathcal{C}$; we shall also refer to them as diagrams.

(vii) Given a category $\mathcal{C}$ and objects $X, Y \in \mathrm{ObC}$, the set of morphisms from $X$ to $Y$ is denoted by $\mathcal{C}(X, Y)$.

(viii) Given a category $\mathcal{C}$, its opposite category is denoted by $\mathcal{C}^{\circ}$.

(ix) A poset $P=(P, \leqslant)=\left(P, \leqslant_{P}\right)$ is a partially ordered set. To consider it as a category, we let $P(p, q)=\{(p \longrightarrow q)\}$ if $p \leqslant q$, and $P(p, q)=\emptyset$ otherwise. A full subposet of a poset is a full subcategory. A subposet is a subcategory. 
(x) A poset $P$ is discrete if $p \leqslant q$ implies $p=q$ for $p, q \in P$; that is, if each morphism in $P$ is an identity.

(xi) Given an exact category $\mathcal{E}$, we denote by $\mathcal{E}^{\text {mono }}$ its subcategory of pure monomorphisms, and by $\mathcal{E}^{\text {epi }}$ its subcategory of pure epimorphisms. By $\rightarrow$, we denote a pure monomorphism; by $\longrightarrow$, we denote a pure epimorphism. Cf. e.g. [6, Sec. A.2].

(xii) A Frobenius category $\mathcal{E}$ is an exact category in which each $X \in \mathrm{Ob} \mathcal{E}$ allows for $N \longrightarrow X \rightarrow N^{\prime}$ with bijective objects $N$ and $N^{\prime}$; cf. e.g. [6, Sec. A.2.3]. Denoting by $\mathcal{B} \subseteq \mathcal{E}$ its full subcategory of bijective objects, we let $\underline{\mathcal{E}}:=\mathcal{E} / \mathcal{B}$ denote the classical stable category of $\mathcal{E}$. Given a morphism $X \stackrel{f}{\longrightarrow} Y$ in $\mathcal{E}$, its residue class in $\underline{\mathcal{E}}$ is denoted by $\underline{X} \stackrel{\underline{f}}{\longrightarrow} \underline{Y}$.

\section{Limits and pure monomorphisms}

\subsection{Crowns}

We extract the relevant part of a poset with respect to taking direct limits of diagrams on it, called its ind-crown, and consider its 1-connectedness.

Definition 1.1. Let $P$ be a finite poset, considered as a category whenever necessary. Given $p \in P$, we define full subposets of $P$

$$
\begin{aligned}
& \Lambda(p)=\Lambda_{P}(p):=\{q \in P: q \leqslant p\} \\
& \mathrm{V}(p)=\mathrm{V}_{P}(p):=\{q \in P: q \geqslant p\} \\
& \Lambda^{0}(p)=\Lambda_{P}^{0}(p):=\{q \in P: q<p\} \\
& \mathrm{V}_{0}(p)=\mathrm{V}_{0, P}(p):=\{q \in P: q>p\} \text {. }
\end{aligned}
$$

Moreover, we define full subposets of $P$

$$
\begin{aligned}
\max (P) & :=\{q \in P: \mathrm{V}(q)=\{q\}\} \\
\min (P) & :=\{q \in P: \Lambda(q)=\{q\}\},
\end{aligned}
$$

which are discrete. We let

$$
\begin{aligned}
& \text { Ob ind-crown }(P):=\bigcup_{p, q \in \max (P)} \operatorname{Ob} \max (\Lambda(p) \cap \Lambda(q)) \\
& \text { Ob pro-crown }(P):=\bigcup_{p, q \in \min (P)} \mathrm{Ob} \min (\mathrm{V}(p) \cap \mathrm{V}(q)) \text {. }
\end{aligned}
$$

The subset $\mathrm{Ob}$ ind-crown $(P)$ of $\mathrm{Ob} P$ carries a structure of a poset by letting

$$
p<_{\text {ind-crown }(P)} q: \Longleftrightarrow p<_{P} q \text { and } p \notin \max (P) \text { and } q \in \max (P)
$$

for $p, q \in \mathrm{Ob}$ ind-crown $(P)$. So ind-crown $(P)$ is a subposet of $P$, but in general not a full subposet of $P$; cf. Example 1.6.

The subset $\mathrm{Ob}$ pro-crown $(P)$ of $\mathrm{Ob} P$ carries a structure of a poset by letting

$$
p<{ }_{\operatorname{pro}-\operatorname{crown}(P)} q: \Longleftrightarrow p<_{P} q \text { and } p \in \min (P) \text { and } q \notin \min (P)
$$

for $p, q \in \mathrm{Ob}$ pro-crown $(P)$. So pro-crown $(P)$ is a subposet of $P$, but in general not a full subposet of $P$.

We have pro-crown $(P)=$ ind-crown $\left(P^{\circ}\right)^{\circ}$. 
A poset $C$ is called a crown if it is finite and if $C=\min (C) \cup \max (C)$. That is, a finite poset $C$ is a crown if there do not exist elements $c, c^{\prime}, c^{\prime \prime} \in C$ with $c<c^{\prime}<c^{\prime \prime}$.

If $P$ is an arbitrary finite poset, then both ind-crown $(P)$ and pro-crown $(P)$ are crowns.

Definition 1.2. Suppose given a crown $C$. Let $\operatorname{Mor}^{\prime} C$ be the set of non identical morphisms of $C$. Let $\mathbf{Q}\left[\operatorname{Mor}^{\prime} C\right]$ be the vector space over $\mathbf{Q}$ with basis $\operatorname{Mor}^{\prime} C$, and let $\mathbf{Q}[\mathrm{Ob} C]$ be the vector space over $\mathbf{Q}$ with basis $\mathrm{Ob} C$.

The crown $C$ is called componentwise 1 -connected if the $\mathbf{Q}$-linear map

$$
\begin{array}{rll}
\mathbf{Q}\left[\operatorname{Mor}^{\prime} C\right] & \stackrel{\partial_{C}}{\longrightarrow} & \mathbf{Q}[\mathrm{Ob} C] \\
(c \longrightarrow d) & \longmapsto & d-c
\end{array}
$$

is injective. Then $C$ is componentwise 1-connected if and only if $C^{\circ}$ is.

In other words, a crown $C$ is componentwise 1-connected if and only if the topological realisation of its nerve is componentwise 1-connected. In fact, for a finite wedge of circles to be 1-connected, i.e. to consist of no circles at all, we may require that $\mathrm{H}^{1}$ vanish.

Lemma 1.3. If $U \subseteq C$ is a full subposet of a componentwise 1-connected crown $C$, then $U$ is itself a componentwise 1-connected crown.

Proof. The poset $U$ is a crown, since there do not exist $c, c^{\prime}, c^{\prime \prime} \in U$ with $c<c^{\prime}<$ $c^{\prime \prime}$, for they do not exist in $C$. By restriction, injectivity of $\mathbf{Q}\left[\mathrm{Mor}^{\prime} C\right] \stackrel{\partial_{C}}{\longrightarrow} \mathbf{Q}[\mathrm{Ob} C]$ implies injectivity of $\mathbf{Q}\left[\mathrm{Mor}^{\prime} U\right] \stackrel{\partial_{U}}{\longrightarrow} \mathbf{Q}[\mathrm{Ob} U]$.

Lemma 1.4 (recursive characterization).

The crown $C$ is componentwise 1-connected if and only if (i) or (ii) or (iii) holds.

(i) There exists $c \in \max (C)$ such that $\# \Lambda^{0}(c) \leqslant 1$, and such that the full subposet $C \backslash\{c\}$ of $C$ is componentwise 1-connected.

(ii) There exists $c \in \min (C)$ such that $\# \mathrm{~V}_{0}(c) \leqslant 1$, and such that the full subposet $C \backslash\{c\}$ of $C$ is componentwise 1-connected.

(iii) $C=\emptyset$.

Proof. Suppose $C \neq \emptyset$ to be componentwise 1-connected. We claim that (i) or (ii) holds.

A chain in $C$ is a tuple $\left(c_{1}, \ldots, c_{m}\right)$ for some $m \geqslant 1$ such that $c_{i}\left\langle c_{i+1}\right.$ or $\left.c_{i}\right\rangle$ $c_{i+1}$ for all $i \in[1, m-1]$, and such that $c_{i+2} \neq c_{i}$ for all $i \in[1, m-2]$. Suppose given such a chain in $C$.

Assume that there are $j, k \in[1, m]$ such that $j<k$, but $c_{j}=c_{k}$. Choose $k-j$ to be minimal with this property. Hence in $\left(c_{j}, c_{j+1}, \ldots, c_{k-1}\right)$, we have pairwise different entries. The number $k-j$ is even and $\geqslant 4$. 
If $c_{j}<c_{j+1}$, then we let

$$
\gamma:=\sum_{i \in[1,(k-j) / 2]}\left(\left(c_{j+2 i-2} \longrightarrow c_{j+2 i-1}\right)-\left(c_{j+2 i} \longrightarrow c_{j+2 i-1}\right)\right) \in \mathbf{Q}\left[\operatorname{Mor}^{\prime} C\right]
$$

if $c_{j}>c_{j+1}$, then we let

$$
\gamma:=\sum_{i \in[1,(k-j) / 2]}\left(\left(c_{j+2 i-1} \longrightarrow c_{j+2 i-2}\right)-\left(c_{j+2 i-1} \longrightarrow c_{j+2 i}\right)\right) \in \mathbf{Q}\left[\operatorname{Mor}^{\prime} C\right] .
$$

In both cases we have $\gamma \neq 0$ since the coefficient of $\left(c_{j} \longrightarrow c_{j+1}\right)$ resp. of $\left(c_{j+1} \longrightarrow c_{j}\right)$ equals 1 . In fact, since $c_{j+1} \neq c_{k-1}$, no cancelation occurs. But $\gamma \partial_{C}=$ 0 , and this contradicts the componentwise 1-connectedness of $C$. From this contradiction we conclude that each chain in $C$ consists of pairwise different entries.

Since $C$ is finite and nonempty, there exists a chain $\left(c_{1}, \ldots, c_{m}\right)$ of maximal length $m$ in $C$. Let $c:=c_{m}$. If $m=1$, then $c$ satisfies both (i) and (ii). So we may suppose $m \geqslant 2$. We claim that $c$ satisfies (i) if $c_{m-1}<c_{m}$, and that $c$ satisfies (ii) if $c_{m-1}>c_{m}$.

Suppose $c_{m-1}<c_{m}$. Assume $\# \Lambda^{0}(c)>1$, and let $c_{m+1} \in \Lambda^{0}(c) \backslash\left\{c_{m-1}\right\}$. Then $\left(c_{1}, \ldots, c_{m-1}, c_{m}, c_{m+1}\right)$ is a chain, contradicting the maximality of $m$. Thus $\# \Lambda^{0}(c) \leqslant 1$. Moreover, $C \backslash\{c\}$ is itself componentwise 1-connected by Lemma 1.3.

Suppose $c_{m-1}>c_{m}$. Assume $\# \mathrm{~V}_{0}(c)>1$, and let $c_{m+1} \in \mathrm{V}_{0}(c) \backslash\left\{c_{m-1}\right\}$. Then $\left(c_{1}, \ldots, c_{m-1}, c_{m}, c_{m+1}\right)$ is a chain, contradicting the maximality of $m$. Thus $\# \mathrm{~V}_{0}(c) \leqslant 1$. Moreover, $C \backslash\{c\}$ is itself componentwise 1-connected by Lemma 1.3.

Conversely, suppose that (i) or (ii) or (iii) holds. We have to show that $C$ is componentwise 1-connected. By duality, we may assume that (i) holds.

If $\Lambda^{0}(c) \neq \emptyset$, we write $\Lambda^{0}(c)=\{d\}$. Then the linear map $\mathbf{Q}\left[\mathrm{Mor}^{\prime} C\right] \stackrel{\partial_{C}}{\longrightarrow} \mathbf{Q}[\mathrm{Ob} C]$ decomposes into

$$
\mathbf{Q}\left[\operatorname{Mor}^{\prime}(C \backslash\{c\})\right] \oplus \mathbf{Q}[\{(d \longrightarrow c)\}] \stackrel{\left(\begin{array}{ccc}
\partial_{C \backslash\{c\}} & 0 \\
-\tilde{d} & \tilde{c}
\end{array}\right)}{\longrightarrow} \mathbf{Q}[\mathrm{Ob}(C \backslash\{c\})] \oplus \mathbf{Q}[\{c\}],
$$

where we denote by $\tilde{d}$ the map that sends $(d \longrightarrow c)$ to $d$, and by $\tilde{c}$ the map that sends $(d \longrightarrow c)$ to $c$.

If $\Lambda^{0}(c)=\emptyset$, then the linear map $\mathbf{Q}\left[\operatorname{Mor}^{\prime} C\right] \stackrel{\partial_{C}}{\longrightarrow} \mathbf{Q}[\mathrm{Ob} C]$ decomposes as

$$
\mathbf{Q}\left[\operatorname{Mor}^{\prime}(C \backslash\{c\})\right] \stackrel{\left(\partial_{C \backslash\{c\}} 0\right)}{\longrightarrow} \mathbf{Q}[\mathrm{Ob}(C \backslash\{c\})] \oplus \mathbf{Q}[\{c\}] .
$$

In both cases, injectivity of $\partial_{C}$ results from injectivity of $\partial_{C \backslash\{c\}}$.

Example 1.5. Let $P=\mathfrak{P}(\{1,2,3\}) \backslash\{\{1,2,3\}\}$, ordered by inclusion. We have $\max (P)=\{\{1,2\},\{1,3\},\{2,3\}\}$. Moreover, we have

$$
\begin{aligned}
& \max (\Lambda(\{1,2\}) \cap \Lambda(\{1,2\}))=\{\{1,2\}\} \\
& \max (\Lambda(\{1,2\}) \cap \Lambda(\{2,3\}))=\{\{2\}\},
\end{aligned}
$$

etc. Thus,

$$
C:=\operatorname{ind}-\operatorname{crown}(P)=\{\{1,2\},\{1,3\},\{2,3\},\{1\},\{2\},\{3\}\} .
$$

In this example, $C$ is actually a full subposet of $P$. The map $\mathbf{Q}\left[\mathrm{Mor}^{\prime} C\right] \stackrel{\partial_{C}}{\longrightarrow} \mathbf{Q}[\mathrm{Ob} C]$, 
$(p \longrightarrow q) \longmapsto q-p$, is given by the matrix

\begin{tabular}{l|rrrrrr} 
& $\{1\}$ & $\{2\}$ & $\{3\}$ & $\{1,2\}$ & $\{1,3\}$ & $\{2,3\}$ \\
\hline$\{1\} \longrightarrow\{1,2\}$ & -1 & 0 & 0 & +1 & 0 & 0 \\
$\{1\} \longrightarrow\{1,3\}$ & -1 & 0 & 0 & 0 & +1 & 0 \\
$\{2\} \longrightarrow\{1,2\}$ & 0 & -1 & 0 & +1 & 0 & 0 \\
$\{2\} \longrightarrow\{2,3\}$ & 0 & -1 & 0 & 0 & 0 & +1 \\
$\{3\} \longrightarrow\{1,3\}$ & 0 & 0 & -1 & 0 & +1 & 0 \\
$\{3\} \longrightarrow\{2,3\}$ & 0 & 0 & -1 & 0 & 0 & +1
\end{tabular}

with kernel $\mathbf{Q}\langle(+1-1-1+1+1-1)\rangle$. Hence the ind-crown $C$ of $P$ is not componentwise 1-connected.

Example 1.6. Let $P=\{\emptyset,\{1\},\{2\},\{2,3\},\{2,4\}\}$, ordered by inclusion. Then Ob ind-crown $(P)=\mathrm{Ob}(P)$. We have $\emptyset<_{P}\{2\}$, however, $\emptyset \nless_{\text {ind-crown }(P)}\{2\}$, since $\{2\} \notin \max (P)$. Thus ind-crown $(P)$ is a subposet of $P$, but not a full subposet. Note that $P$ is not a crown, but that, of course, ind-crown $(P)$ is a crown.

Example 1.7. Let $P=\{\{1\},\{2\},\{1,2\},\{2,3\}\}$, ordered by inclusion. Then $P$ is a crown. We have

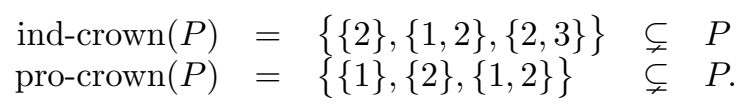

\subsection{Limits}

We generalise familiar properties of pushouts in exact categories to direct limits over more general diagrams.

Let $\mathcal{E}$ be an exact category; cf. e.g. [6, Sec. A.2]. Let $P$ be a poset. Given a diagram $X \in \mathrm{Ob} \mathcal{E}(P)$, we write $X_{p}:=X(p)$ for $p \in \mathrm{Ob} P$, and $\xi_{p, q}:=X(p \longrightarrow q)$ whenever $p, q \in \mathrm{Ob} P$ with $p \leqslant q$. We write $\lim _{P} X=\lim _{\longrightarrow} X_{P} X_{p}$. Similarly, the morphisms in a diagram $X^{\prime} \in \mathrm{Ob} \mathcal{E}(P)$ are denoted by $\xi_{p, q}^{\prime}$, etc.

Lemma 1.8. Let $C$ be a componentwise 1-connected crown, and let $X \in \mathrm{Ob} \mathcal{E}(C)$ be a diagram consisting of pure monomorphisms $\xi_{c, d}$ for all $c, d \in C$ with $c \leqslant d$. Then $\lim _{C} X$ exists, and the transition morphism $X_{c} \longrightarrow \lim _{C} X$ is a pure monomorphism for each $c \in C$.

Proof. We may assume that $C \neq \emptyset$. We proceed by induction on $\# C$ and choose $c \in C$ such that condition (i) or (ii) of Lemma 1.4 holds. Let $L:=\left.\lim _{\longrightarrow \backslash \backslash\{c\}} X\right|_{C \backslash\{c\}}$, with transition morphism $X_{e} \stackrel{\eta_{e}}{\rightarrow} L$ for $e \in C \backslash\{c\}$.

Consider the case that condition (i) of Lemma 1.4 holds for $c$. If $\Lambda^{0}(c)=\emptyset$, then $\lim _{\longrightarrow} X=L \oplus X_{c}$, and the transition morphisms are given by $X_{e} \stackrel{\left(\eta_{e} 0\right)}{\rightarrow} L \oplus X_{c}$ for $\overrightarrow{e \in C} C \backslash\{c\}$ and by $X_{c} \stackrel{(01)}{\rightarrow} L \oplus X_{c}$.

If $\Lambda^{0}(c)$ consists of one element, say $\Lambda^{0}(c)=\{d\}$, then we consider the pushout 


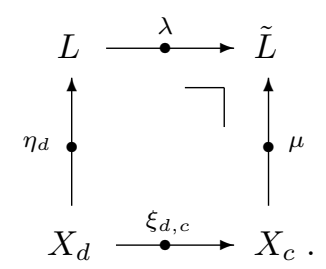

We have $\lim _{C} X=\tilde{L}$, and the transition morphisms are given by $X_{e} \stackrel{\eta_{e} \lambda}{\rightarrow} \tilde{L}$ for $e \in C \backslash\{c\}$ and by $X_{c} \stackrel{\mu}{\rightarrow} \tilde{L}$.

Consider the case that condition (ii) of Lemma 1.4 holds for $c$. We may assume that $\mathrm{V}_{0}(c)$ consists of one element, say $\mathrm{V}_{0}(c)=\{d\}$, for otherwise condition (i) holds. We have $\lim _{\longrightarrow} X=L$, and the transition morphisms are given by $X_{e} \stackrel{\eta_{e}}{\rightarrow} L$ for $e \in C \backslash\{c\}$ and by $X_{c} \stackrel{\xi_{c, d} \eta_{d}}{\rightarrow} L$.

Example 1.9. Let $C=\{\{1\},\{2\},\{1,2,3\},\{1,2,4\}\}$, ordered by inclusion; the poset $C$ is not componentwise 1-connected. Let $a:=\{1\}, b:=\{2\}, u:=\{1,2,3\}$ and $v:=\{1,2,4\}$. Let $\mathcal{E}=\mathbf{Z}$-mod be the category of finitely generated $\mathbf{Z}$-modules, with all short exact sequences being pure short exact. Let $X_{a}=X_{b}=X_{u}=X_{v}=\mathbf{Z}$, let $\xi_{a, u}=1, \xi_{a, v}=1, \xi_{b, u}=1$ and $\xi_{b, v}=m \geqslant 2$. Then $\lim _{C} X=\mathbf{Z} /(m-1)$, with transition morphisms $X_{u} \stackrel{1}{\rightarrow} \mathbf{Z} /(m-1)$ and $X_{v} \stackrel{1}{\rightarrow} \mathbf{Z} /(m-1)$. The diagram $X$ consists of pure monomorphisms. But none of the transition morphisms to the limit is a pure monomorphism.

Proposition 1.10. Suppose given a finite poset $P$ such that $C:=\operatorname{ind}-\operatorname{crown}(P)$ is componentwise 1-connected. Suppose given a diagram $X \in \mathrm{Ob} \mathcal{E}(P)$ with $\xi_{p, q}$ purely monomorphic for all $p, q \in \mathrm{Ob} P$. The following assertions (i, ii) hold.

(i) The limits $\left.\lim _{C} X\right|_{C}$ and $\lim _{P} X$ exist in $\mathcal{E}$, and the canonical morphism

$$
\left.\underset{C}{\lim _{P}} X\right|_{C} \longrightarrow \underset{P}{\lim } X
$$

is an isomorphism.

(ii) The transition morphism $X_{p} \longrightarrow \lim _{P} X$ is a pure monomorphism for $p \in P$.

Proof. By Lemma 1.8, it suffices to prove that, with transition morphisms defined by composition, $L:=\left.\lim _{C} X\right|_{C}$ is the direct limit of the whole diagram $X$. Denote by $X_{c} \stackrel{\eta_{c}}{\rightarrow} L$ the transition morphism for $c \in C$.

So for $p \in P$, as transition morphism from $X_{p}$ to $L$ we take

$$
\left(X_{p} \stackrel{\vartheta_{p}}{\rightarrow} L\right):=\left(X_{p} \stackrel{\xi_{p, c}}{\rightarrow} X_{c} \stackrel{\eta_{c}}{\rightarrow} L\right)
$$

for some $c \in \max (P) \subseteq C$ such that $p \leqslant c$. We need to show that this definition does not depend on the choice of $c$. So assume given $d \in \max (P) \backslash\{c\}$ such that $p \leqslant d$. We have to show that $\xi_{p, c} \eta_{c}=\xi_{p, d} \eta_{d}$. Note that $p \in \Lambda(c) \cap \Lambda(d)$. Let $e \in$ $\max (\Lambda(c) \cap \Lambda(d)) \subseteq C$. Then $e \notin \max (P)$, hence $e<_{C} c$ and $e<_{C} d$. Thus we obtain

$$
\xi_{p, c} \eta_{c}=\xi_{p, e} \xi_{e, c} \eta_{c}=\xi_{p, e} \eta_{e}=\xi_{p, e} \xi_{e, d} \eta_{d}=\xi_{p, d} \eta_{d}
$$

As to the universal property of the direct limit, suppose given a family of morphisms 
$\left(X_{p} \stackrel{\zeta_{p}}{\longrightarrow} Z\right)_{p \in P}$ such that $\xi_{p, q} \zeta_{q}=\zeta_{p}$ whenever $p, q \in P$ such that $p \leqslant q$. We obtain an induced morphism $L \stackrel{\zeta}{\longrightarrow} Z$ such that $\eta_{c} \zeta=\zeta_{c}$ for $c \in C$. Uniqueness of $\zeta$ is already given with respect to $C$, so it will hold a fortiori with respect to $P$. It remains to show the existence with respect to $P$, that is, that $\vartheta_{p} \zeta=\zeta_{p}$ for $p \in P$. In fact, using an element $c \in \max (P)$ with $p \leqslant c$, we obtain

$$
\vartheta_{p} \zeta=\xi_{p, c} \eta_{c} \zeta=\xi_{p, c} \zeta_{c}=\zeta_{p} .
$$

\section{Replacement lemmata}

\subsection{Replacement}

Definition 2.1. A finite poset $D$ is called ind-flat if ind-crown $\left(\Lambda^{0}(d)\right)$ is componentwise 1-connected for each $d \in D$. Dually, $D$ is called pro-flat if pro-crown $\left(\mathrm{V}_{0}(d)\right)$ is componentwise 1-connected for each $d \in D$. Altogether, $D$ is called flat if $D$ is ind-flat and pro-flat.

\section{Example 2.2.}

(i) The poset $P$ in Example 1.5 is ind-flat. It is not pro-flat, since

$$
\operatorname{pro-crown}\left(\mathrm{V}_{0}(\emptyset)\right)=\{\{1\},\{2\},\{3\},\{1,2\},\{1,3\},\{2,3\}\}
$$

is not componentwise 1-connected.

(ii) The poset $P$ in Example 1.6 is flat.

(iii) The poset $P$ in Example 1.7 is flat.

(iv) The poset $\Delta_{m} \times \Delta_{n}$ is flat for $m, n \geqslant 0$.

(v) The poset $\{\emptyset,\{1\},\{2\},\{3\},\{1,4\},\{1,5\},\{1,2,3\},\{3,4\},\{3,5\},\{1,2,3,4,5\}\}$ is flat.

(vi) The poset $\Delta_{1} \times \Delta_{1} \times \Delta_{1} \simeq \mathfrak{P}(\{1,2,3\})$ is neither ind-flat nor pro-flat.

(vii) More generally, the poset $\Delta_{1}^{m} \simeq \mathfrak{P}([1, m])$ is neither ind-flat nor pro-flat for $m \geqslant 3$.

Example 2.3. If $D$ is a flat finite poset and $D^{\prime} \subseteq D$ a full subposet, then $D^{\prime}$ is not ind-flat in general.

For instance, let $D=\{\{1\},\{2\},\{1,2\},\{1,2,3\},\{1,2,4\},\{1,2,3,4\}\}$, containing the full subposet $D^{\prime}=\{\{1\},\{2\},\{1,2,3\},\{1,2,4\},\{1,2,3,4\}\}$. Then $D$ is flat. In $D^{\prime}$, however, ind-crown $\left(\Lambda_{D^{\prime}}^{0}(\{1,2,3,4\})\right)=\{\{1\},\{2\},\{1,2,3\},\{1,2,4\}\}$ is not componentwise 1-connected, and so $D^{\prime}$ is not ind-flat.

Suppose given a Frobenius category $\mathcal{E}$; cf. e.g. [6, Sec. A.2.3]. Suppose given a finite poset $D$.

Definition 2.4. A prefunctor $X$ from $D$ to $\mathcal{E}$ assigns to each object $a$ of $D$ an object $X_{a}$ of $\mathcal{E}$, and to each morphism $a \longrightarrow b$ of $D$ a morphism $\xi_{a, b}$ of $\mathcal{E}$ in such a way that whenever $a \leqslant b \leqslant c$ in $D$, then $\xi_{a, b} \xi_{b, c}-\xi_{a, c}$ is homotopic to zero, i.e. it factors over a bijective object in $\mathcal{E}$. Sometimes, we refer to $X$ as a prediagram on $D$ with values in $\mathcal{E}$. 
Given prefunctors $X$ and $X^{\prime}$ from $D$ to $\mathcal{E}$, a morphism $X^{\prime} \stackrel{f}{\longrightarrow} X$ is a tuple $\left(X_{a}^{\prime} \stackrel{f_{a}}{\longrightarrow} X_{a}\right)_{a \in \mathrm{Ob} D}$ such that $f_{a} \xi_{a, b}=\xi_{a, b}^{\prime} f_{b}$ whenever $a \leqslant b$ in $D$. Such a morphism $X^{\prime} \stackrel{f}{\longrightarrow} X$ is called a homotopism if its image $\underline{X}^{\prime} \stackrel{f}{\longrightarrow} \underline{X}$ in $\underline{\mathcal{E}}(D)$ is an isomorphism.

Let $\mathcal{E}^{\sim}(D)$ be the category of prefunctors from $D$ to $\mathcal{E}$. In particular, a homotopism is a morphism in $\mathcal{E}^{\sim}(D)$. We have a full subcategory $\mathcal{E}(D) \subseteq \mathcal{E}^{\sim}(D)$ consisting of diagrams - a diagram is in particular a prediagram.

There is a canonical dense functor $\mathcal{E}^{\sim}(D) \longrightarrow \underline{\mathcal{E}}(D), X \longmapsto \underline{X}$, given by taking residue classes of the morphisms of $X$.

Remark 2.5. Suppose given $X \in \mathrm{Ob}^{\sim}(D)$, a bijective object $N$ in $\mathcal{E}$ and $a \in D$. Let $X^{\prime} \in \mathrm{Ob} \mathcal{E}^{\sim}(D)$ be such that

$$
\begin{aligned}
X_{b}^{\prime} & = \begin{cases}X_{b} & \text { if } b \neq a \\
X_{a} \oplus N & \text { if } b=a\end{cases} \\
\left(X_{b}^{\prime} \stackrel{\xi_{b, c}^{\prime}}{\longrightarrow} X_{c}^{\prime}\right) & = \begin{cases}X_{b} \stackrel{\xi_{b, c}}{\longrightarrow} X_{c} & \text { if } b<c \text { and } a \notin\{b, c\} \\
X_{a} \oplus N \stackrel{\left(\begin{array}{c}
\xi_{a, c} \\
\eta_{c}
\end{array}\right)}{\longrightarrow} X_{c} & \text { if } a=b<c \\
X_{b} \stackrel{\left(\begin{array}{l}
\xi_{b, a} \\
\zeta_{b}
\end{array}\right)}{\longrightarrow} X_{a} \oplus N & \text { if } b<c=a,\end{cases}
\end{aligned}
$$

for some $N \stackrel{\eta_{c}}{\longrightarrow} X_{c}$ for $c \in \mathrm{V}_{0}(a)$ and some $X_{b} \stackrel{\zeta_{b}}{\longrightarrow} N$ for $b \in \Lambda^{0}(a)$. We call $X^{\prime}$ a replacement of $X$ at $a \in D$.

There is an isomorphism

$$
\begin{aligned}
\underline{X}^{\prime} & \stackrel{\sim}{X_{b}} \stackrel{\frac{X}{1}}{\longrightarrow} X_{b} \quad \text { if } b \neq a \\
X_{a} \oplus N & \stackrel{\left(\begin{array}{l}
1 \\
0
\end{array}\right)}{\longrightarrow} X_{a}
\end{aligned}
$$

in $\underline{\mathcal{E}}(D)$. If $a \in \max (D)$, this isomorphism lifts to a homotopism $X^{\prime} \longrightarrow X$ in $\mathcal{E}^{\sim}(D)$.

\subsection{A purely monomorphic replacement}

Lemma 2.6. Suppose given a finite poset $D$ and an element $c \in \max (D)$. Suppose ind-crown $\left(\Lambda^{0}(c)\right)$ to be componentwise 1-connected.

Suppose given a diagram $X \in \mathrm{Ob} \mathcal{E}(D)$ such that $\left.X\right|_{D \backslash\{c\}} \in \mathrm{Ob}^{\mathrm{mono}}(D \backslash\{c\})$, i.e. such that its restriction to $D \backslash\{c\}$ consists of pure monomorphisms. Then there exist $X^{\prime} \in \mathrm{Ob} \mathcal{E}^{\text {mono }}(D)$ and a homotopism $X^{\prime} \stackrel{f}{\longrightarrow} X$.

Proof. Let $L:=\left.\lim _{\Lambda^{0}(c)} X\right|_{\Lambda^{0}(c)}$, which exists in $\mathcal{E}$ by Proposition 1.10.(i). Let $X_{b} \stackrel{\eta_{b}}{\rightarrow} L$ denote the transition morphism for $b \in \Lambda^{0}(c)$, which is purely monomorphic by Proposition 1.10.(ii). Let $L \stackrel{\zeta}{\longrightarrow} X_{c}$ be the unique morphism such that 
$\eta_{b} \zeta=\xi_{b, c}$ for all $b \in \Lambda^{0}(c)$. Choose a pure monomorphism $L \stackrel{\iota}{\rightarrow} N$ with $N$ bijective. For a replacement at $c$ in the sense of Remark 2.5, we let $X_{c}^{\prime}:=X_{c} \oplus N$ and

$$
\left(X_{b}^{\prime} \stackrel{\xi_{b, c}^{\prime}}{\longrightarrow} X_{c}^{\prime}\right):=\left(X_{b} \stackrel{\left(\xi_{b, c} \eta_{b} \iota\right)}{\longrightarrow} X_{c} \oplus N\right)
$$

for $b \in \Lambda^{0}(c)$. This yields a diagram $X^{\prime} \in \operatorname{Ob} \mathcal{E}^{\operatorname{mono}}(D)$. Since $c \in \max (D)$, Remark 2.5 gives a homotopism $X^{\prime} \longrightarrow X$.

Lemma 2.7. Given a ind-flat finite poset $D$ and a diagram $X \in \operatorname{Ob} \mathcal{E}(D)$. Then there exist $X^{\prime} \in \mathrm{Ob}^{\text {mono }}(D)$ and a homotopism $X^{\prime} \stackrel{f}{\longrightarrow} X$.

Proof. We proceed by induction on $\# D$ and may assume $\# D \geqslant 1$. Let $c \in \max (D)$. Since $D \backslash\{c\}$ is ind-flat, too, we may assume the assertion to hold for the diagram $\left.X\right|_{D \backslash\{c\}}$ on $D \backslash\{c\}$; i.e. we may assume there exists a homotopism $\left.Y \stackrel{g}{\longrightarrow} X\right|_{D \backslash\{c\}}$ in $\mathcal{E}(D \backslash\{c\})$ for some $Y \in \mathrm{Ob}^{\mathrm{mono}}(D \backslash\{c\})$. Define $X^{\prime \prime} \in \mathrm{Ob} \mathcal{E}(D)$ by

$$
\begin{array}{lll}
\left.X^{\prime \prime}\right|_{D \backslash\{c\}} & = \\
X_{c}^{\prime \prime} & =X_{c} \\
\left(X_{b}^{\prime \prime} \stackrel{\xi_{b, c}^{\prime}}{\longrightarrow} X_{c}^{\prime \prime}\right) & =\left(Y_{b} \stackrel{g_{b}}{\longrightarrow} X_{b} \stackrel{\xi_{b, c}}{\longrightarrow} X_{c}\right) \text { for } b \in D \backslash\{c\} .
\end{array}
$$

In $\mathcal{E}(D)$, we have a homotopism $X^{\prime \prime} \stackrel{f}{\longrightarrow} X$ given by

$$
\begin{aligned}
& \left(X_{b}^{\prime \prime} \stackrel{f_{b}}{\longrightarrow} X_{b}\right)=\left(Y_{b} \stackrel{g_{b}}{\longrightarrow} X_{b}\right) \quad \text { for } b \in D \backslash\{c\} \\
& \left(X_{c}^{\prime \prime} \stackrel{f_{c}}{\longrightarrow} X_{c}\right)=\left(X_{c} \stackrel{1_{X_{c}}}{\longrightarrow} X_{c}\right) .
\end{aligned}
$$

Finally, by Lemma 2.6 , we can replace $X^{\prime \prime}$ by an object $X^{\prime}$ in $\mathcal{E}^{\text {mono }}(D)$.

\subsection{A replacement that adds a commutativity}

Lemma 2.8. Suppose given a finite poset $D$, an element $c \in \max (D)$, an element $d \in \max \left(\Lambda^{0}(c)\right)$, and an element $e \in \Lambda^{0}(d)$. So $e<d<c$, and there is no element between $d$ and c. Suppose ind-crown $\left(\Lambda^{0}(d)\right)$ to be componentwise 1-connected.

Suppose given $X \in \mathrm{Ob}^{\sim}(D)$ such that (I, II) hold.

(I) We have $\left.X\right|_{D \backslash\{c\}} \in \operatorname{Ob} \mathcal{E}(D \backslash\{c\})$.

(II) We have $\left.X\right|_{\Lambda^{0}(c)} \in \operatorname{Ob} \mathcal{E}^{\text {mono }}\left(\Lambda^{0}(c)\right)$.

Then there exist $X^{\prime} \in \mathrm{Ob}^{\sim}(D)$ and an isomorphism $\underline{X}^{\prime} \stackrel{\sim}{\longrightarrow} \underline{X}$ in $\underline{\mathcal{E}}(D)$ such that (i, ii, iii, iv) hold.

(i) We have $\left.X^{\prime}\right|_{D \backslash\{c\}} \in \operatorname{Ob} \mathcal{E}(D \backslash\{c\})$.

(ii) We have $\left.X^{\prime}\right|_{\Lambda^{0}(c)} \in \operatorname{Ob} \mathcal{E}^{\text {mono }}\left(\Lambda^{0}(c)\right)$.

(iii) We have $\xi_{e, c}^{\prime}=\xi_{e, d}^{\prime} \xi_{d, c}^{\prime}$.

(iv) We have $\left.\left.X^{\prime}\right|_{D \backslash\{d\}} \simeq X\right|_{D \backslash\{d\}}$ in $\mathcal{E}^{\sim}(D \backslash\{d\})$.

Proof. Denote $L:=\left.\underline{\lim }_{\Lambda^{0}(d)} X\right|_{\Lambda^{0}(d)}$, and let $X_{b} \stackrel{\eta_{b}}{\rightarrow} L$ be the transition morphism for $b \in \Lambda^{0}(d)$; cf. Proposition 1.10. Let $L \stackrel{\zeta}{\longrightarrow} X_{d}$ be the unique morphism such that $\eta_{b} \zeta=\xi_{b, d}$ for all $b \in \Lambda^{0}(d)$. Choose a pure monomorphism $L \stackrel{\iota}{\rightarrow} N$ with $N$ bijective. 
Bijectivity of $N$ together with pure monomorphy of $\eta_{e} \iota$ allows the nullhomotopic difference $\xi_{e, c}-\xi_{e, d} \xi_{d, c}$ to be factored as

$$
\xi_{e, c}-\xi_{e, d} \xi_{d, c}=\eta_{e} \iota \vartheta
$$

for some $N \stackrel{\vartheta}{\longrightarrow} X_{c}$.

For a replacement at $d$ in the sense of Remark 2.5, we let $X_{d}^{\prime}:=X_{d} \oplus N$ and

$$
\begin{array}{lll}
\left(X_{b}^{\prime} \stackrel{\xi_{b, d}^{\prime}}{\longrightarrow} X_{d}^{\prime}\right):=\left(X_{b} \stackrel{\left(\xi_{b, d} \eta_{b \iota}\right)}{\longrightarrow} X_{d} \oplus N\right) & \text { for } b \in \Lambda^{0}(d) \\
\left(X_{d}^{\prime} \stackrel{\xi_{d, c}^{\prime}}{\longrightarrow} X_{c}^{\prime}\right):=\left(X_{d} \oplus N \stackrel{\left(\begin{array}{c}
\xi_{d, c} \\
\vartheta
\end{array}\right)}{\longrightarrow} X_{c}\right) & \\
\left(X_{d}^{\prime} \stackrel{\xi_{d, a}^{\prime}}{\longrightarrow} X_{a}^{\prime}\right):=\left(X_{d} \oplus N \stackrel{\left(\begin{array}{c}
\xi_{d, a} \\
0
\end{array}\right)}{\longrightarrow} X_{a}\right) & \text { for } a \in \mathrm{V}_{0}(d) \backslash\{c\} .
\end{array}
$$

This yields the required diagram $X^{\prime}$.

\section{Density}

Theorem 3.1. Suppose given an ind-flat finite poset D. Then the residue class functor

$$
\begin{aligned}
\mathcal{E}^{\text {mono }}(D) & \longrightarrow \underline{\mathcal{E}}(D) \\
X & \longmapsto \underline{X}
\end{aligned}
$$

is dense.

Proof. We proceed by induction on $\# D$. We may assume $\# D \geqslant 1$. Let $c \in \max (D)$. Suppose given $X \in \mathrm{Ob} \mathcal{E}^{\sim}(D)$. Since $D \backslash\{c\}$ is ind-flat, by induction, there exists a diagram $Y \in \operatorname{Ob}^{\text {mono }}(D \backslash\{c\})$ such that $\underline{Y} \stackrel{g}{\sim} \underline{X}$. Extending $Y$ to a diagram $\hat{Y} \in \operatorname{Ob} \mathcal{E}^{\sim}(D)$ by appending $X_{c}$ at $c$, and morphisms $Y_{d} \stackrel{\hat{g}_{d} \xi_{d, c}}{\longrightarrow} X_{c}$ for $d<c$, where $\hat{g}_{d}$ is a representative of $g_{d}$, we obtain $\underline{\hat{Y}} \simeq \underline{X}$ via an isomorphism that restricts to $g$ on $D \backslash\{c\}$ and to the identity on $\{c\}$. Moreover, $\left.\hat{Y}\right|_{D \backslash\{c\}} \in \operatorname{Ob}^{\text {mono }}(D)$. So we may assume that $\left.X\right|_{D \backslash\{c\}} \in \mathrm{Ob}^{\text {mono }}(D)$.

A full subposet $U \subseteq$ ind-crown $\left(\Lambda^{0}(c)\right.$ ) is called commutant (with respect to $X$ ) whenever there exist $X^{\prime} \in \mathrm{Ob} \mathcal{E}^{\sim}(D)$ and an isomorphism $\underline{X}^{\prime} \stackrel{\sim}{\longrightarrow} \underline{X}$ such that (1), (2) and (3) hold.

(1) We have $\left.X^{\prime}\right|_{D \backslash\{c\}} \in \operatorname{Ob} \mathcal{E}(D \backslash\{c\})$.

(2) We have $\left.X^{\prime}\right|_{\Lambda^{0}(c)} \in \operatorname{Ob} \mathcal{E}^{\text {mono }}\left(\Lambda^{0}(c)\right)$.

(3) We have $\xi_{s, t}^{\prime} \xi_{t, c}^{\prime}=\xi_{s, c}^{\prime}$ for all $s, t \in U$ with $s<t$.

By assumption, ind-crown $\left(\Lambda^{0}(c)\right)$ is componentwise 1-connected, so by Lemma 1.3, any full subposet $U \subseteq$ ind-crown $\left(\Lambda^{0}(c)\right)$ is a componentwise 1-connected crown, too.

We claim that each full subposet $U \subseteq$ ind-crown $\left(\Lambda^{0}(c)\right)$ is commutant.

We perform an induction on $\# U$. We may assume $\# U \geqslant 1$. By Lemma 1.4, we can distinguish the following two cases.

Case (i). There exists $u \in \max (U)$ such that $\# \Lambda_{U}^{0}(u) \leqslant 1$. If $\Lambda_{U}^{0}(u)=\emptyset$, then we conclude from $U \backslash\{u\}$ being commutant that $U$ is commutant. So suppose 
that, say, $\Lambda_{U}^{0}(u)=\{v\}$. By induction, we may assume that $\xi_{s, t} \xi_{t, c}=\xi_{s, c}$ for all $s, t \in U \backslash\{u\}$ with $s<t$. We use Lemma 2.8 in the following way. In the notation used there, we let $c=c, d=u$ and $e=v$, and get an $X^{\prime} \in \mathrm{Ob} \mathcal{E}^{\sim}(D)$ and an isomorphism $\underline{X}^{\prime} \stackrel{\sim}{\longrightarrow}$ such that $\xi_{s, t}^{\prime} \xi_{t, c}^{\prime}=\xi_{s, c}^{\prime}$ for all $s, t \in U \backslash\{u\}$ with $s<t$ by Lemma 2.8 (iv), and such that $\xi_{v, u}^{\prime} \xi_{u, c}^{\prime}=\xi_{v, c}^{\prime}$ by loc. cit. (iii). Finally, $\left.X^{\prime}\right|_{D \backslash\{c\}} \in$ $\mathrm{Ob} \mathcal{E}(D \backslash\{c\})$ by loc. cit. (i) and $\left.X^{\prime}\right|_{\Lambda^{0}(c)} \in \mathrm{Ob} \mathcal{E}^{\text {mono }}\left(\Lambda^{0}(c)\right)$ by loc. cit. (ii). Thus $U$ is commutant.

Case (ii). There exists $u \in \min (U)$ such that $\# \mathrm{~V}_{0, U}(u) \leqslant 1$. If $\mathrm{V}_{0, U}(u)=\emptyset$, then we conclude from $U \backslash\{u\}$ being commutant that $U$ is commutant. So suppose that, say, $\mathrm{V}_{0, U}(u)=\{v\}$. By induction, we may assume that $\xi_{s, t} \xi_{t, c}=\xi_{s, c}$ for all $s, t \in U \backslash\{u\}$ with $s<t$. We define $X^{\prime} \in \mathrm{Ob} \mathcal{E}^{\sim}(D)$ by letting $\xi_{s, t}^{\prime}:=\xi_{s, t}$ if $s, t \in$ $D$ with $s<t$ and $(s, t) \neq(u, c)$, and letting $\xi_{u, c}^{\prime}:=\xi_{u, v} \xi_{v, c}=\xi_{u, v}^{\prime} \xi_{v, c}^{\prime}$. Then $\underline{X}^{\prime}=$ $\underline{X}$ and $\xi_{s, t}^{\prime} \xi_{t, c}^{\prime}=\xi_{s, c}^{\prime}$ for all $s, t \in U$ with $s<t$. Moreover, $\left.X^{\prime}\right|_{D \backslash\{c\}}=\left.X\right|_{D \backslash\{c\}} \in$ $\mathrm{Ob} \mathcal{E}(D \backslash\{c\})$ and $\left.X^{\prime}\right|_{\Lambda^{0}(c)}=\left.X\right|_{\Lambda^{0}(c)} \in \mathrm{Ob}^{\text {mono }}\left(\Lambda^{0}(c)\right)$. Thus $U$ is commutant.

This proves the claim. In particular, ind-crown $\left(\Lambda^{0}(c)\right)$ is commutant, and we dispose of an according diagram $X^{\prime} \in \mathrm{Ob} \mathcal{E}^{\sim}(D)$ satisfying (1), (2) and (3).

Now define $X^{\prime \prime} \in \mathrm{Ob} \mathcal{E}^{\sim}(D)$ by letting $\xi_{b, d}^{\prime \prime}:=\xi_{b, d}^{\prime}$ for $b<d \neq c$ and $\xi_{b, c}^{\prime \prime}:=\xi_{b, t}^{\prime} \xi_{t, c}^{\prime}$ for $b \in \Lambda^{0}(c)$, for some $t \in \max \left(\Lambda^{0}(c)\right)$ with $b \leqslant t$. Since $\xi_{s, t}^{\prime} \xi_{t, c}^{\prime}=\xi_{s, c}^{\prime}$ for all $s, t \in$ ind-crown $\left(\Lambda^{0}(c)\right)$ with $s<t$, this definition of $\xi_{b, c}^{\prime}$ does not depend on the choice of $t$, and we have in fact $X^{\prime \prime} \in \operatorname{Ob} \mathcal{E}(D)$ with $\underline{X}^{\prime \prime}=\underline{X}^{\prime}$.

By Lemma 2.7, there exist $X^{\prime \prime \prime} \in \mathrm{Ob}^{\text {mono }}(D)$ and a homotopism $X^{\prime \prime \prime} \longrightarrow X^{\prime \prime}$.

Scholium 3.2. Given a flat finite poset $D$, the residue class functors $\mathcal{E}^{\text {mono }}(D) \longrightarrow$ $\underline{\mathcal{E}}(D)$ and $\mathcal{E}^{\mathrm{epi}}(D) \longrightarrow \underline{\mathcal{E}}(D)$ are dense.

Example 3.3. We claim that given $X \in \mathrm{Ob} \mathcal{E}^{\sim}(D)$, in general there do not exist $X^{\prime} \in \mathrm{Ob} \mathcal{E}(D)$ and a homotopism $X^{\prime} \longrightarrow X$.

Given a finite poset $D$ such that $D \times \Delta_{1}$ is ind-flat, this failure prevents us from using density of $\mathcal{E}^{\text {mono }}\left(D \times \Delta_{1}\right) \longrightarrow \underline{\mathcal{E}}\left(D \times \Delta_{1}\right)$ together with [6, Lem. A.35] to conclude that $\mathcal{E}^{\text {mono }}(D) \longrightarrow \underline{\mathcal{E}}(D)$ is 1-epimorphic.

Proof of the claim. Let $D=\Delta_{2}$. Let $\mathcal{E}$ be a Frobenius category in which not every object is bijective. Let $X \in \mathrm{Ob} \mathcal{E}^{\sim}(D)$ be defined to have a non-bijective object $X_{0}$, an arbitrary object $X_{1}$ and a bijective object $X_{2}$ such that there exists $X_{0} \stackrel{i}{\rightarrow} X_{2}$; and by morphisms $\xi_{0,1}=0, \xi_{1,2}=0$ and $\xi_{0,2}=i$.

Assume there is a homotopism $X^{\prime} \longrightarrow X$ for some $X^{\prime} \in \mathrm{Ob} \mathcal{E}(D)$, consisting of morphisms $X_{i}^{\prime} \stackrel{u_{i}}{\longrightarrow} X_{i}$ for $i \in[0,2]$. Then $u_{1} \xi_{1,2}=\xi_{1,2}^{\prime} u_{2}$ shows that $\xi_{1,2}^{\prime} u_{2}=0$. Hence

$$
u_{0} i=u_{0} \xi_{0,2}=\xi_{0,2}^{\prime} u_{2}=\xi_{0,1}^{\prime} \xi_{1,2}^{\prime} u_{2}=0 .
$$

Since $i$ is monomorphic, this implies $u_{0}=0$. Since $u_{0}$ is an isomorphism, we conclude that $X_{0} \simeq 0$, i.e. that $X_{0}$ is bijective, contradicting our assumption. Thus there does not exist a homotopism $X^{\prime} \longrightarrow X$ with $X^{\prime} \in \mathrm{Ob} \mathcal{E}(D)$. 
Question 3.4. Is there a poset $D$ and a Frobenius category $\mathcal{E}$ such that the residue class functor $\mathcal{E}^{\text {mono }}(D) \longrightarrow \mathcal{E}(D)$ is not dense? What about, say, $D=\Delta_{1} \times \Delta_{1} \times$ $\Delta_{1}$ ? Is there a counterexample if we relax the condition on $D$ and allow $D$ to be an arbitrary finite category?

To illustrate the kind of problem addressed in Question 3.4, we briefly report a failed attempt to find a counterexample.

Example 3.5. We let the finite category $D$ be defined by $\mathrm{Ob} D=\{c\}$ and by ${ }_{D}(c, c)=\left\{1_{c}, \alpha\right\}$, where $\alpha \neq 1_{c}$, but $\alpha^{2}=1_{c}$. Let $X:=(C \stackrel{a}{\longrightarrow} C)$ be an endomorphism of $\mathcal{E}$ that is an object of $\mathcal{E}^{\sim}(D)$, i.e. assume $a^{2}-1$ to vanish in $\underline{\mathcal{E}}$. Let $C \stackrel{u}{\rightarrow} N$ be a pure monomorphism into a bijective object. Consider a factorization $a^{2}-1=u v$ and a prolongation $N \stackrel{\tilde{a}}{\longrightarrow} N$ of $a$ along $u$, i.e. $u \tilde{a}=a u$. Note that $u(\tilde{a} v-v a)=0$ and $u\left(\tilde{a}^{2}-1-v u\right)=0$.

Assume that $u, v$ and $\tilde{a}$ can be chosen such that the following hold.

(1) We have $\tilde{a} v-v a=0$.

(2) We have $\tilde{a}^{2}-1-v u=0$.

For example, we might take $\mathcal{E}=\mathbf{Z} / 27-\bmod , C=\mathbf{Z} / 9, a=2, N=\mathbf{Z} / 27, u=3$, $v=1$ and $\tilde{a}=2$.

Let $X^{\prime} \in \mathrm{Ob} \mathcal{E}(D)$ be defined by $C \oplus N \stackrel{\left(\begin{array}{cc}a & u \\ -v & -\tilde{a}\end{array}\right)}{\longrightarrow} C \oplus N$. Then $\underline{X} \simeq \underline{X}^{\prime}$ in $\underline{\mathcal{E}}(D)$ via $C \stackrel{(10)}{\longrightarrow} C \oplus N$. So in order to find a counterexample in this manner, it is necessary to use an endomorphism $a$ for which, for all choices of $v$ and $\tilde{a}$, condition (1) or (2) fails.

\section{1-Epimorphy}

Definition 4.1. A finite poset $D$ is called a quasi-tree if for all $a, b \in D$, the full subposet $\mathrm{V}_{0}(a) \cap \Lambda^{0}(b)$ of $D$ is linearly ordered.

Example 4.2. Suppose given a finite poset $D$.

(i) If $D$ is a crown, then it is a quasi-tree, since then $\mathrm{V}_{0}(a) \cap \Lambda^{0}(b)=\emptyset$ for all $a, b \in D$.

(ii) If for $a, b \in D$ such that $a \not b$ and $a \ngtr b$, we have $\mathrm{V}(a) \cap \mathrm{V}(b)=\emptyset$, then the poset $D$ is called an ascending tree. An ascending tree is a quasi-tree.

(iii) The poset $D$ is a quasi-tree if and only if its full subposet $\mathrm{V}(a)$ is an ascending tree for all $a \in D$.

Lemma 4.3. Suppose given a finite poset D. The following are equivalent.

(i) The poset $D$ is a finite quasi-tree.

(ii) The subposet ind-crown $\left(\Lambda^{0}(a)\right)$ of $D$ is discrete for all $a \in D$.

(iii) The subposet pro-crown $\left(\mathrm{V}^{0}(a)\right)$ of $D$ is discrete for all $a \in D$.

In particular, if $D$ is a finite quasi-tree, then $D$ is flat. 
Proof. First of all, we remark that ind-crown $\left(\Lambda^{0}(a)\right)$ is discrete if and only if ind-crown $\left(\Lambda^{0}(a)\right)=\max \left(\Lambda^{0}(a)\right)$, i.e. if and only if

$$
\Lambda^{0}(b) \cap \Lambda^{0}\left(b^{\prime}\right)=\emptyset
$$

for all $b, b^{\prime} \in \max \left(\Lambda^{0}(a)\right)$ with $b \neq b^{\prime}$.

Ad (i) $\Longrightarrow$ (ii). Suppose given $b, b^{\prime} \in \max \left(\Lambda^{0}(a)\right)$ with $b \neq b^{\prime}$. Assume there exists $c \in \Lambda^{0}(b) \cap \Lambda^{0}\left(b^{\prime}\right)$. Then $b, b^{\prime} \in \mathrm{V}_{0}(c) \cap \Lambda^{0}(a)$, but $b \nless b^{\prime}$ and $b \ngtr b^{\prime}$ because of their maximality in $\Lambda^{0}(a)$. But $\mathrm{V}_{0}(c) \cap \Lambda^{0}(a)$ is linearly ordered. This contradiction shows that $\Lambda^{0}(b) \cap \Lambda^{0}\left(b^{\prime}\right)=\emptyset$.

Ad (ii) $\Longrightarrow$ (i). Given $a, c \in D$, we have to show that $\mathrm{V}_{0}(c) \cap \Lambda^{0}(a)$ is linearly ordered. Assume there exist $b$ and $b^{\prime}$ in $\mathrm{V}_{0}(c) \cap \Lambda^{0}(a)$ such that $b \notin b^{\prime}$ and $b \ngtr b^{\prime}$. Choose $d \in \min \left(\Lambda(a) \cap \mathrm{V}_{0}(b) \cap \mathrm{V}_{0}\left(b^{\prime}\right)\right)$. Choose $e \in \max \left(\mathrm{V}(b) \cap \Lambda^{0}(d)\right)$. Choose $e^{\prime} \in \max \left(\mathrm{V}\left(b^{\prime}\right) \cap \Lambda^{0}(d)\right)$. Then $e$ and $e^{\prime}$ are different elements of $\max \left(\Lambda^{0}(d)\right)$, because $e=e^{\prime}$ would imply $e \notin\left\{b, b^{\prime}\right\}$, and we could replace $d$ by $e$, contradicting the minimality of $d$. We have $e, e^{\prime} \in \max \left(\Lambda^{0}(d)\right)$, whereas

$$
c \in \Lambda^{0}(e) \cap \Lambda^{0}\left(e^{\prime}\right) \neq \emptyset,
$$

which is impossible by (ii). This contradiction shows that $\mathrm{V}_{0}(c) \cap \Lambda^{0}(a)$ is in fact linearly ordered.

A functor $\mathcal{U} \stackrel{F}{\leftarrow} \mathcal{V}$ is called 1-epimorphic if the induced functor $\mathcal{C}(\mathcal{U}) \stackrel{\mathcal{C}(F)}{\longrightarrow} \mathcal{C}(\mathcal{V})$, given by restriction along $F$, is full and faithful for any category $\mathcal{C}$; cf. [6, Sec. A.8].

Proposition 4.4. Suppose given a finite quasi-tree D. Then the residue class functor

$$
\begin{aligned}
\mathcal{E}^{\text {mono }}(D) & \longrightarrow \underline{\mathcal{E}}(D) \\
X & \longmapsto \underline{X}
\end{aligned}
$$

is 1-epimorphic.

Proof. By Lemma 4.3 and Theorem 3.1, this functor is dense. So by [6, Lem. A.35],

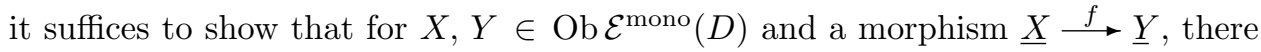
exist a homotopism $X^{\prime} \stackrel{g^{\prime}}{\longrightarrow} X$ and a morphism $X^{\prime} \stackrel{g}{\longrightarrow} Y$ in $\mathcal{E}(D)$ such that

$$
\left(\underline{X}^{\prime} \stackrel{\underline{g}^{\prime}}{\longrightarrow} \underline{X} \stackrel{f}{\longrightarrow} \underline{Y}\right)=\left(\underline{X}^{\prime} \stackrel{g}{\longrightarrow} \underline{Y}\right) .
$$

The morphisms that $X$ consists of are denoted by $\xi_{a, b}$, the morphisms that $Y$ consists of by $\eta_{a, b}$, etc., where $a, b \in D$ with $a<b$.

We proceed by induction on $\# D$. We may assume $\# D \geqslant 1$. Let $c \in \max (D)$. By induction, the assertion holds for $D \backslash\{c\}$. Letting $X_{c}^{\prime \prime}:=X_{c}$, by composition, we obtain a diagram $X^{\prime \prime} \in \mathrm{Ob} \mathcal{E}(D)$, a homotopism $X^{\prime \prime} \stackrel{h^{\prime}}{\longrightarrow} X$ and a morphism $\left.\left.X^{\prime \prime}\right|_{D \backslash\{c\}} \stackrel{h}{\longrightarrow} Y\right|_{D \backslash\{c\}}$ such that the following hold.

(i) The diagram $\left.X^{\prime \prime}\right|_{D \backslash\{c\}}$ is in $\mathrm{Ob}^{\text {mono }}(D \backslash\{c\})$.

(ii) We have $\left.\left.\underline{h}^{\prime}\right|_{D \backslash\{c\}} f\right|_{D \backslash\{c\}}=\underline{h}$.

(iii) We have $h_{c}^{\prime}=1_{X_{c}}$. 
We choose a representative $X_{c}^{\prime \prime} \stackrel{\hat{f}_{c}}{\longrightarrow} Y_{c}$ in $\mathcal{E}$ of $f_{c}$. We choose a pure monomorphism

$$
\bigoplus_{a \in \max \left(\Lambda^{0}(c)\right)} X_{a} \stackrel{\left(i_{a}\right)_{a}}{\longrightarrow} N
$$

into a bijective object $N$. In particular, each $i_{a}$ is purely monomorphic. We have a factorisation

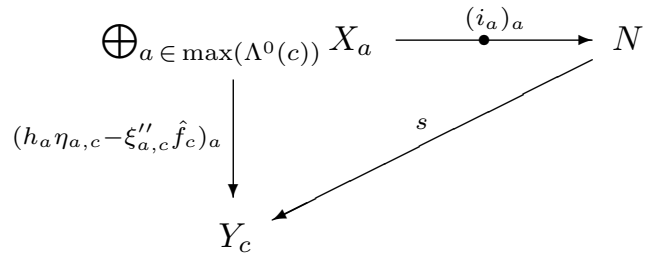

Define a replacement $X^{\prime}$ of $X^{\prime \prime}$ at $c$ in the sense of Remark 2.5 by $X_{c}^{\prime}:=X_{c}^{\prime \prime} \oplus N$ and by

$$
\left(X_{b}^{\prime} \stackrel{\xi_{b, c}^{\prime}}{\longrightarrow} X_{c}^{\prime}\right):=\left(X_{b}^{\prime \prime} \stackrel{\left(\xi_{b, a}^{\prime \prime} \xi_{a, c}^{\prime \prime} \stackrel{\xi_{b, a}^{\prime \prime} i_{a}}{\longrightarrow}\right)}{\longrightarrow} X_{c} \oplus N\right)
$$

for $b \in \Lambda^{0}(c)$, where $\{a\}=\max \left(\mathrm{V}(b) \cap \Lambda^{0}(c)\right)$, which is well defined since $D$ is a quasi-tree. Then $X^{\prime} \in \mathrm{Ob} \mathcal{E}^{\text {mono }}(D)$. Let $X^{\prime} \stackrel{h^{\prime \prime}}{\longrightarrow} X^{\prime \prime}$ be the homotopism of Remark 2.5 , and let $\left(X^{\prime} \stackrel{g^{\prime}}{\longrightarrow} X\right):=\left(X^{\prime} \stackrel{h^{\prime \prime}}{\longrightarrow} X^{\prime \prime} \stackrel{h^{\prime}}{\longrightarrow} X\right)$. Let $X^{\prime} \stackrel{g}{\longrightarrow} Y$ be defined by

$$
\left\{\begin{array}{lll}
\left(X_{b}^{\prime} \stackrel{g_{b}}{\longrightarrow} Y_{b}\right) & :=\left(X_{b}^{\prime \prime} \stackrel{h_{b}}{\longrightarrow} Y_{b}\right) & \text { at } b \neq c \\
\left(X_{c}^{\prime} \stackrel{g_{c}}{\longrightarrow} Y_{c}\right) & :=\left(X_{c} \oplus N \stackrel{\left(\begin{array}{c}
\hat{f}_{c} \\
s
\end{array}\right)}{\longrightarrow} Y_{c}\right) & \text { at } c .
\end{array}\right.
$$

We claim that $g^{\prime} f=g$. If $b \neq c$, we obtain

$$
\left(\underline{g}^{\prime} f\right)_{b}=\underline{h}_{b}^{\prime} f_{b}=\underline{h}_{b}=\underline{g}_{b} .
$$

At $c$, we obtain

$$
\left(\underline{g}^{\prime} f\right)_{c}=\left(\begin{array}{l}
1 \\
0
\end{array}\right) f_{c}=\left(\begin{array}{c}
f_{c} \\
0
\end{array}\right)=\underline{\left(\begin{array}{c}
\hat{f}_{c} \\
s
\end{array}\right)}=\underline{g}_{c}
$$

Scholium 4.5. Given a finite quasi-tree $D$, the residue class functors $\mathcal{E}^{\text {mono }}(D) \longrightarrow$ $\underline{\mathcal{E}}(D)$ and $\mathcal{E}^{\mathrm{epi}}(D) \longrightarrow \underline{\mathcal{E}}(D)$ are dense and 1-epimorphic.

Using Lemma 4.3, this summarises Scholium 3.2, Proposition 4.4 and its dual assertion in the given situation.

Example 4.6. We claim that given a finite quasi-tree $D$, the residue class functor $\mathcal{E}^{\text {mono }}(D) \longrightarrow \underline{\mathcal{E}}(D)$ is not full in general.

A full and dense functor is 1-epimorphic; cf. [6, Cor. A.37]. This example, together with Scholium 4.5, shows that this implication is strict.

Proof of the claim. Let $D=\{\{1\},\{2\},\{1,2\}\}$, let $p \geqslant 3$ be a prime, and let $\mathcal{E}=$ $\mathbf{Z} / p^{3}$-mod, with all short exact sequences being purely short exact. An object is bijective if and only if it is a finite direct sum of copies of $\mathbf{Z} / p^{3}$. Consider the following morphism in $\underline{\mathcal{E}}(D)$. 


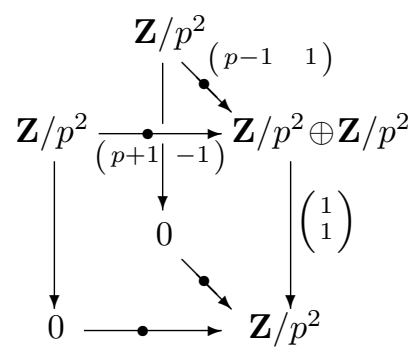

The question of whether it lifts to a morphism in $\mathcal{E}^{\text {mono }}(D)$ is equivalent to the question of whether there exist $h, k \in \mathbf{Z} / p$ such that

$$
\begin{aligned}
& \left(\begin{array}{ll}
p-1 & 1
\end{array}\right)\left(\left(\begin{array}{l}
1 \\
1
\end{array}\right)+p\left(\begin{array}{l}
h \\
k
\end{array}\right)\right) \equiv_{p^{2}} 0 \\
& \left(\begin{array}{ll}
p+1 & -1
\end{array}\right)\left(\left(\begin{array}{l}
1 \\
1
\end{array}\right)+p\left(\begin{array}{l}
h \\
k
\end{array}\right)\right) \equiv_{p^{2}} 0 .
\end{aligned}
$$

Adding the two resulting equations, we get $2 p \equiv_{p^{2}} 0$, so we cannot find the required $h$ and $k$.

Question 4.7. Given an ind-flat finite poset $D$ and a Frobenius category $\mathcal{E}$, is the residue class functor $\mathcal{E}^{\text {mono }}(D) \longrightarrow \underline{\mathcal{E}}(D)$ then 1-epimorphic?

\section{Work of Cooke, Dwyer-Kan-Smith and Mitchell}

Let $G$ be a group, considered as a category. By a space we mean a topological space homotopy equivalent to a $\mathrm{CW}$-complex. Let (Spaces) be the category of spaces and continuous maps. Let (Hot) be the category of spaces and homotopy classes of continuous maps.

Cooke developed in [1] an obstruction theory for the induced functor

$$
\llbracket G,(\text { Spaces }) \rrbracket \longrightarrow \llbracket G,(\text { Hot }) \rrbracket
$$

to be dense. The obstructions are classes in the cohomology groups of $G$ with certain coefficients in dimensions $\geqslant 3$; cf. [1, Th. 1.1].

DwYER, KAN and SMith generalised this obstruction theory in [2] from a group $G$ to an arbitrary category $D$ (and even to topological categories). The obstruction to the density of the according functor are then classes in the Hochschild-Mitchell groups of $D$ with certain coefficients in dimensions $\geqslant 3$, and a problem "involving fundamental groupoids"; cf. [2, 3.5,3.6].

Mitchell has given in [8] the following criterion for the Hochschild-Mitchell cohomological dimension of a poset to be less than or equal to 2 .

Given $n \geqslant 2$, the suspended $n$-crown $\mathrm{SC}_{n}$ is the poset defined as follows. As a set, let $\mathrm{SC}_{n}:=\left\{u_{i}, v_{i}: i \in \mathbf{Z} / n\right\} \sqcup\{s, t\}$ consist of $2 n+2$ elements. The partial ordering on $\mathrm{SC}_{n}$ is generated by

$$
v_{i}<u_{i}, \quad v_{i}<u_{i-1}, \quad u_{i}<t \text { and } s<v_{i} \text { for all } i \in \mathbf{Z} / n .
$$

Suppose given a finite poset D. According to [8, Th. 35.7], its Hochschild-Mitchell cohomology vanishes in dimensions $\geqslant 3$ for all $D$-bimodules as coefficients if and 
only if neither (i) nor (ii) holds.

(i) The poset $D$ contains an isomorphic copy of $\mathrm{SC}_{n}$ as a full subposet for some $n \geqslant 3$.

(ii) The poset $D$ contains an isomorphic copy of $\mathrm{SC}_{2}$ as a full subposet, and there is no $d \in D$ such that $v_{0} \leqslant d, v_{1} \leqslant d, d \leqslant u_{0}$ and $d \leqslant u_{1}$.

Question 5.1. Suppose a finite poset satisfies condition (i) or (ii). Does it follow that it is not ind-flat?

I do not know a counterexample. An affirmative answer would hint at the possible existence of obstruction classes in certain Hochschild-Mitchell cohomology groups in dimension $\geqslant 3$ to the density of

$$
\llbracket D, \mathcal{E} \rrbracket \longrightarrow \llbracket D, \mathcal{E} \rrbracket
$$

for an arbitrary Frobenius category $\mathcal{E}$. Ind-flat finite posets would then have vanishing Hochschild-Mitchell cohomology in dimension $\geqslant 3$, so, provided such an obstruction theory exists, we would see the "real reason" why an ind-flat finite poset $D$ yields a dense functor $(*)$; cf. Theorem 3.1 .

The following two simple examples should point out problems one might possibly encounter when trying to approach Question 5.1.

Example 5.2. Let

$$
D:=\{\emptyset,\{1\},\{2\},\{3\},\{1,2\},\{2,3\},\{1,3\},\{1,2,3\},\{1,3,4\},\{1,2,3,4\}\},
$$

ordered by inclusion.

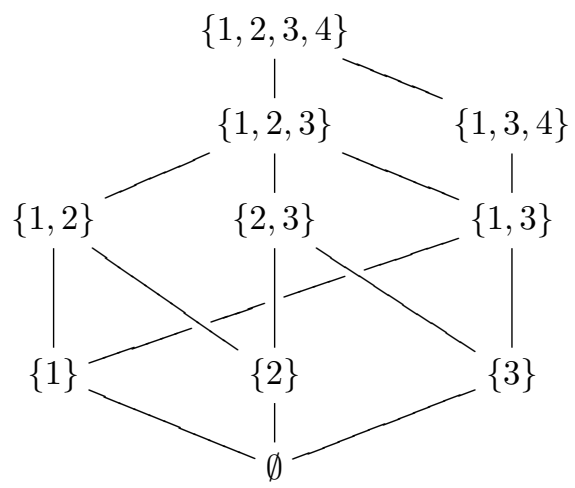

It contains the suspended 3-crown $D \backslash\{\{1,2,3\},\{1,3\}\}$ as a full subposet. Moreover, $\{1,2,3,4\}$ is a minimal element in $\mathrm{V}(\{1,2\}) \cap \mathrm{V}(\{2,3\}) \cap \mathrm{V}(\{1,3,4\})$. However, ind-crown $\left(\Lambda^{0}(\{1,2,3,4\})\right)$ is componentwise 1-connected. Only ind-crown $\left(\Lambda^{0}(\{1,2,3\})\right)$ is not.

Thus, if we are given a finite poset that contains a suspended 3-crown with maximal element $t$, and even if, moreover, $t$ is chosen to be minimal with respect to lying over the rest of the suspended 3-crown, we can still not conclude that ind-crown $\left(\Lambda^{0}(t)\right)$ is not componentwise 1-connected. Instead, we will have to search 
elsewhere for a suitable element $t^{\prime}$ such that ind-crown $\left(\Lambda^{0}\left(t^{\prime}\right)\right)$ is not componentwise 1-connected in order to prove failure of ind-flatness.

Example 5.3. Let

$$
D:=\{\emptyset,\{1\},\{2\},\{3\},\{1,2\},\{2,3,4\},\{1,3,4\},\{1,2,3\},\{1,2,3,4\}\},
$$

ordered by inclusion.

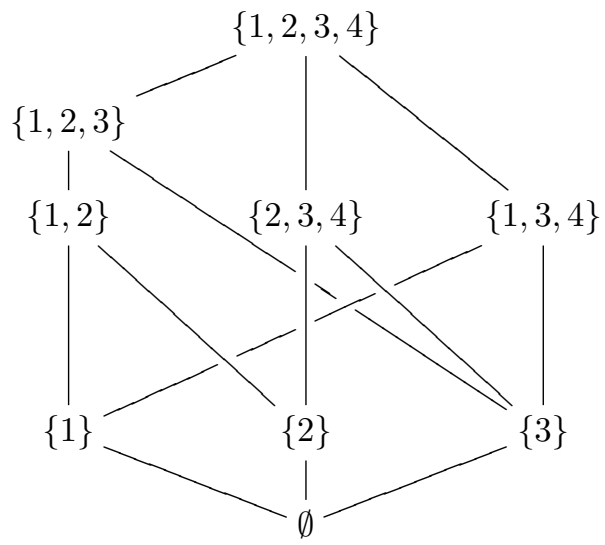

It contains the suspended 3-crown $D^{\prime}:=D \backslash\{\{1,2,3\}\}$ as a full subposet. Now ind-crown $\left(\Lambda_{D^{\prime}}^{0}(\{1,2,3,4\})\right)$ is homotopy equivalent to a circle, whereas ind-crown $\left(\Lambda_{D}^{0}(\{1,2,3,4\})\right)$ is homotopy equivalent to a wedge of two circles. So $D$ is not ind-flat. The reason for this, however, is an ind-crown of a somewhat surprising shape.

Thus if we want to attach some kind of homotopical invariant to a poset, or to a pair consisting of a poset and an element of it, and if we want to prove that this invariant is preserved under certain full embeddings of posets, we are confronted with this "jump phenomenon".

\section{References}

[1] Cooke, G., Replacing homotopy actions by topological actions, Trans. Am. Math. Soc. 237, (1978) 391-406.

[2] Dwyer, W. G., Kan, D. M. and Smith, J. H., Homotopy commutative diagrams and their realizations, J. Pure Appl. Alg. 57, (1989) 5-24.

[3] Grothendieck, A., Les Dérivateurs, www.math.jussieu.fr/ maltsin/groth/Derivateurs.html, 1990.

[4] Heller, A., Homotopy theories, Mem. Am. Math. Soc. 383, 1988.

[5] Keller, B., Le dérivateur triangulé associé à une catégorie exacte, manuscript, www.math.jussieu.fr/ maltsin/Gtder.html, 2001.

[6] KÜNZER, M., Heller triangulated categories, preprint, math.CT/0508565, 2005. 
[7] Maltsiniotis, G., La K-théorie d'un dérivateur triangulé, preprint, www.math.jussieu.fr/ maltsin, 2002.

[8] Mitchell, B., Rings with several objects, Adv. Math. 8, (1972) 1-161.

[9] Quillen, D., Higher algebraic K-theory: I, Lecture Notes in Mathematics, 341, Springer-Verlag, Berlin, 1973.

[10] Verdier, J. L., Catégories dérivées (état 0), SGA 4 1/2, Lecture Notes in Mathematics, 569, (1977) 262-311, (written 1963).

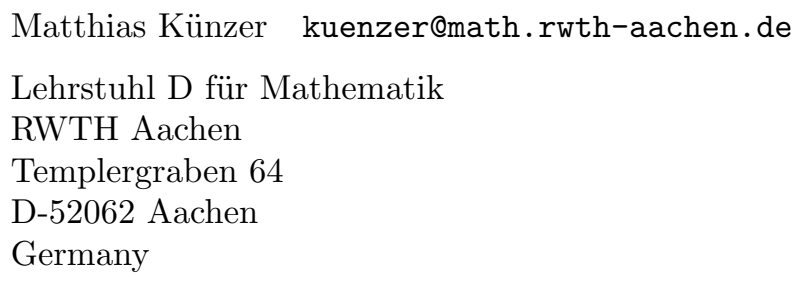

This article is available at http://intlpress.com/HHA/v8/n2/a6 